\section{Frequency of Occult High-Grade Squamous Intraepithelial Neoplasia and Invasive Cancer within Anal Condylomata in Men Who Have Sex with Men}

\begin{abstract}
Hans P. Schlecht, ${ }^{1}$ Dana K. Fugelso, ${ }^{2}$ Ryan K. Murphy, Katiri T. Wagner, ${ }^{5}$ John P. Doweiko, ${ }^{3}$ JoAnn Proper, ${ }^{6}$ Bruce J. Dezube, $^{3}$ and Lori A. Panther, ${ }^{4,5}$

'Department of Medicine, Division of Infectious Diseases and HIV Medicine, Drexel College of Medicine, Philadelphia, Pennsylvania; ${ }^{2}$ Department of Surgery and ${ }^{3}$ Department of Internal Medicine, Division of Hematology/Oncology, and ${ }^{4}$ Department of Internal Medicine, Division of Infectious Diseases, Beth Israel Deaconess Medical Center, Harvard Medical School, and ${ }^{5}$ The Fenway Institute, Fenway Community Health, Boston, Massachusetts; ${ }^{6}$ Department of Medicine, Division of Medical Oncology, Duke Comprehensive Cancer Center, Duke University School of Medicine, Durham, North Carolina; and 'University of New England College of Osteopathic Medicine, Biddeford, Maine
\end{abstract}

Human papillomavirus causes anal condylomata, high-grade anal intraepithelial neoplasia, and anal squamous cell cancer. We found high-grade intraepithelial neoplasia or squamous cell cancer in $75(47 \%)$ of 159 HIV-seropositive men who have sex with men (MSM) and in $42(26 \%)$ of $160 \mathrm{HIV}-$ seronegative MSM with anal condylomata meriting surgery $\left(P<.001\right.$, determined by use of the $\chi^{2}$ test $)$. Anal condylomata in MSM often harbor high-grade intraepithelial neoplasia and squamous cell cancer.

The 2 main groups of human papillomavirus (HPV) types that infect the anogenital tract are low-risk HPV types, which are associated with the development of anogenital condylomata, and high-risk HPV types, which are implicated in the evolution of anal squamous cell cancer and its putative precursor, highgrade anal intraepithelial neoplasia [1]. Repeated exposure, infection, and shedding of genital HPV are common and overlapping events in sexually active populations, and simultaneous detection of multiple HPV types in the genital tract is a recognized phenomenon, especially in immunosuppressed pop-

Received 3 December 2009; accepted 16 March 2010; electronically published 19 May 2010 .

Reprints or correspondence: Dr Lori A. Panther, The Fenway Institute, 1340 Boylston, Boston, MA 02215 (Ipanther@bidmc.harvard.edu).

Clinical Infectious Diseases 2010;51(1):107-110

(C) 2010 by the Infectious Diseases Society of America. All rights reserved. 1058-4838/2010/5101-0018\$15.00

DOI: $10.1086 / 653426$ ulations [2]. HIV-related immunosuppression may increase the chances of both low-grade and high-grade HPV lesions coexisting in the same patient [3].

The rate of anal squamous cell cancer in HIV-seropositive men who have sex with men (MSM) has increased in the decades following the institution of highly active antiretroviral therapy, suggesting that longer life expectancies may permit established high-risk HPV infections to progress to anal squamous cell cancer in a small proportion of patients [4]. Reports of histopathological findings in anogenital condylomata in large MSM populations are limited. In a study by McCloskey et al [5], at least one-third of 74 MSM and over half of $26 \mathrm{HIV}-$ seropositive MSM had high-grade anal intraepithelial neoplasia within surgically excised warts. We report the prevalence of occult high-grade anal intraepithelial neoplasia and anal squamous cell cancer in a large urban cohort of MSM with anal condylomata too extensive to be amenable to outpatient ablation.

Methods. A retrospective cohort of all MSM referred to a single university-affiliated surgical practice for ablation of anal condylomata from May 2002 through April 2007 was evaluated. Patients were referred from their primary provider or from the affiliated high-resolution anoscopy clinic for evaluation of anogenital warts deemed too widely distributed and/or too large to undergo office treatment. High-resolution anoscopy was used in the operating room to identify all lesions, which were then surgically excised using a $\mathrm{CO}_{2}$ laser. All excised tissue was submitted for histopathological analysis. Histopathologic grade was recorded as anal intraepithelial neoplasia grade 1 , anal intraepithelial neoplasia grade 2 or grade 3 (high-grade anal intraepithelial neoplasia), or anal squamous cell cancer.

The protocol for electronic medical record review underwent formal evaluation and waiver by the Beth Israel Deaconess Medical Center Committee on Clinical Investigations. Subjects with a history of anal high-grade anal intraepithelial neoplasia or anal squamous cell cancer were excluded. Subjects with documented HIV infection were recorded as HIV seropositive, and those with either laboratory documentation or absence of HIV infection on the electronic medical record problem list were recorded as HIV seronegative. If available, presenting symptoms and anal cytology results within 6 months prior to surgery were documented. In HIV-positive subjects, the results of CD4 cell count and HIV viral load within 6 months of surgery were recorded.

Anal cytology specimens were collected from the transformation zone of the anal canal by use of moistened Dacron 
swabs and then fixed in liquid ThinPrep (Cytyc) solution. Results were reported, per the 2001 Bethesda classification system, as follows: normal cells; atypical squamous cells of undetermined significance; atypical squamous cells (cannot exclude high-grade squamous intraepithelial lesions); low-grade squamous intraepithelial lesions; and high-grade squamous intraepithelial lesions [6].

Data were stratified by HIV serostatus and histologic grade. Categorical variables were compared between groups by use of the Fisher exact test or the $\chi^{2}$ test when appropriate, and normally distributed continuous data were compared by use of the 1-way analysis of covariance with multiple comparisons or the 2 -tailed unpaired $t$ test. For nonparametric comparisons, the Spearman rank correlation test or the Kruskal-Wallis test was used. CD4 cell counts were $\log _{10}$-transformed, with a nondetectable viral load recorded as 50 copies $/ \mathrm{mL}$. Sensitivity and specificity of anal cytology for detection of high-grade anal intraepithelial neoplasia or anal squamous cell cancer were calculated (GraphPad InStat for Windows, version 3.06; GraphPad Software, available at http://www.graphpad.com).

Results. Table 1 summarizes the clinical characteristics of and the histopathologic findings for the 319 subjects included in the analysis. The cohort was fairly evenly divided between HIV-seropositive subjects $(n=159)$ and HIV-seronegative subjects $(n=160)$. Most subjects were referred from their primary providers, and more HIV-seropositive subjects were referred from the high-resolution anoscopy clinic than were HIV- seronegative subjects. The mean age of the entire cohort was 37 years (range, 18-73 years), and the HIV-seropositive group was significantly older than the HIV-seronegative group $(P<$ .001 , determined by use of an unpaired $t$ test with Welch correction). Except for the greater proportion of HIV-seronegative subjects presenting with pain $(P=.02$, determined by use of the Fisher exact test), presenting symptoms had similar frequencies regardless of HIV serostatus. There was no significant trend toward increasing frequency of symptoms with worsening histologic grade (data not shown).

Of the 319 subjects in the cohort, 202 (63\%) had anal intraepithelial neoplasia grade 1 on histopathological exam, 109 (34\%) had high-grade anal intraepithelial neoplasia, and 8 (3\%) had anal squamous cell cancer. High-grade anal intraepithelial neoplasia or anal squamous cell cancer was present in 75 (47\%) of 159 HIV-seropositive subjects and in 42 (26\%) of $160 \mathrm{HIV}$ seronegative subjects $\left(P=.002\right.$, determined by use of the $\chi^{2}$ test). Seven of the 8 subjects with anal squamous cell cancer were HIV seropositive. Subjects with anal squamous cell cancer were older (mean age, 51 years; range, 35-73 years) than subjects with anal intraepithelial neoplasia grade 1 (mean age, 36 years; range, 18-69 years) or subjects with high-grade anal intraepithelial neoplasia (mean age, 38 years; range, 19-71 years) ( $P=.002$, determined by use of the Kruskal-Wallis test).

Anal cytology findings were available for 123 (39\%) of 319 subjects, and there was correlation between anal cytology and histopathology grades $\left(P=.001\right.$, determined by use of the $\chi^{2}$

Table 1. Age, Presenting Characteristics, and Histopathological Findings in Men Who Have Sex with Men (MSM) with Anal Condylomata Meriting Surgical Excision

\begin{tabular}{|c|c|c|c|c|}
\hline Characteristic & $\begin{array}{c}\text { Total } \\
(n=319)\end{array}$ & $\begin{array}{l}\text { HIV-seropositive MSM } \\
\qquad(n=159)\end{array}$ & $\begin{array}{l}\text { HIV-seronegative MSM } \\
\qquad(n=160)\end{array}$ & $P^{\mathrm{a}}$ \\
\hline \multicolumn{5}{|l|}{ Age } \\
\hline Mean years $\pm S D$ & $37(10)$ & $41(8)$ & $34(11)$ & $<.001$ \\
\hline Median years (IQR) & $37(30-44)$ & $40(36-45)$ & $32(24-40)$ & $<.001$ \\
\hline \multicolumn{5}{|l|}{ MSM reporting symptoms } \\
\hline Pain & $75 / 286(26)$ & $29 / 145(20)$ & $46 / 141(33)$ & .02 \\
\hline Palpable mass & $234 / 286(82)$ & $115 / 141(82)$ & $119 / 145(82)$ & NS \\
\hline Bleeding & $117 / 286(41)$ & $58 / 141(41)$ & $59 / 145(41)$ & NS \\
\hline Itching & $58 / 286(20)$ & $31 / 141(22)$ & 27/145 (19) & NS \\
\hline Referral source & & & & $<.001$ \\
\hline Primary care provider & $209 / 319(65)$ & 78/159 (49) & 131/160 (82) & \\
\hline High-resolution anoscopy clinic & $110 / 319(35)$ & $81 / 159(51)$ & 29/160 (18) & \\
\hline Histological grade & & & & .002 \\
\hline Anal intraepithelial neoplasia grade 1 & $202 / 319(63)$ & $84 / 159(53)$ & $118 / 160(74)$ & \\
\hline High-grade anal intraepithelial neoplasia ${ }^{b}$ & $109 / 319(34)$ & $68 / 159(43)$ & $41 / 160(26)$ & \\
\hline Anal squamous cell cancer & 8/319 (3) & $7 / 159$ (4) & $1 / 160(1)$ & \\
\hline
\end{tabular}

NOTE. Data are proportion (\%) of MSM, unless otherwise indicated. IQR, interquartile range; NS, not significant; SD, standard deviation.

a $P$ value (2-sided) for unpaired $t$ test with Welch correction (mean values), Mann-Whitney test (median values), Fisher exact test, or $\chi^{2}$ test for independence comparing HIV-seropositive and HIV-seronegative subjects.

${ }^{\mathrm{b}}$ Includes anal intraepithelial neoplasia grade 2 and anal intraepithelial neoplasia grade 3. 
test). A finding of a high-grade squamous intraepithelial lesion or an atypical squamous cell (cannot exclude high-grade squamous intraepithelial lesion) on anal cytology correlated with the presence of high-grade anal intraepithelial neoplasia or anal squamous cell cancer on histopathology $(P=.002$, determined by use of the Fisher exact test). This association remained statistically significant in the HIV-seropositive group $(P<.001$, determined by use of the Fisher exact test) but not in the HIVseronegative group ( $P=.353$, determined by use of the Fisher exact test). The sensitivity for high-grade squamous intraepithelial lesions on anal cytology for presence of high-grade anal intraepithelial neoplasia or anal squamous cell cancer on histopathology was 90\% (95\% confidence interval [CI], 81\%96\%) over the entire cohort, 93\% (95\% CI, 83\%-98\%) for HIV-seropositive subjects, and 89\% (95\% CI, 73\%-98\%) for HIV-seronegative subjects. However, the specificity for highgrade squamous intraepithelial lesions on anal cytology for high-grade anal intraepithelial neoplasia or anal squamous cell cancer was only $33 \%$ (95\% CI, 21\%-48\%) over the entire cohort, 58\% (95\% CI, 37\%-78\%) for HIV-seropositive MSM, and 23\% (95\% CI, 5\%-54\%) for HIV-seronegative MSM.

CD4 cell counts and/or HIV viral loads within 6 months of surgery were available for $127(80 \%)$ of the 159 HIV-seropositive subjects. Overall, the mean CD4 cell count was $431 \pm$ 238 cells $/ \mathrm{mm}^{3}$ (range, $4-1153$ cells $/ \mathrm{mm}^{3}$ ), and 60 (50\%) of 121 subjects had detectable viral loads (range, 75-523,000 copies/ $\mathrm{mL}$ ). There was a trend toward correlation between CD4 cell count and the $\log _{10}$ (HIV viral load) that did not reach statistical significance $(P=.08$, determined by use of the Pearson correlation test). There was no significant overall correlation between either CD4 cell count or HIV viral load and histopathologic grade. The mean CD4 cell count ( \pm standard deviation) of the $7 \mathrm{HIV}$-positive subjects with anal squamous cell cancer was lower than that of the rest of the group $(218 \pm 124$ cells/ $\mathrm{mm}^{3}$ vs $441 \pm 237$ cells $/ \mathrm{mm}^{3} ; P=.02$, determined by use of an unpaired $t$ test). There was no correlation between HIV viral load and histopathologic grade.

Discussion. Because anogenital condylomata are classically associated with low-risk HPV types [7], the presence of highgrade anal intraepithelial neoplasia within what clinically appears to be a low-grade condylomatous lesion is unsettling. In this large observational cohort of MSM undergoing surgical excision of anogenital condylomata, 118 (37\%) of 319 subjects demonstrated high-grade anal intraepithelial neoplasia or anal squamous cell cancer, with HIV-seropositive MSM approximately twice as likely to harbor high-grade anal intraepithelial neoplasia or anal squamous cell cancer, compared with HIVseronegative MSM (75 [47\%] of 159 HIV-seropositive MSM vs 42 [26\%] of $160 \mathrm{HIV}$-seronegative MSM). Smaller cohorts of MSM have reported high-grade anal intraepithelial neoplasia rates in the range of $16 \%-71 \%$ in HIV-seropositive populations and in the range of $6 \%-46 \%$ in $\mathrm{HIV}$-seronegative populations [8-10].

Our study shows the sensitivity and specificity of high-grade squamous intraepithelial lesions on preoperative anal cytology for detection of high-grade anal intraepithelial neoplasia or anal squamous cell cancer, which are similar to the sensitivity and specificity that we previously reported for the detection of highgrade anal intraepithelial neoplasia by high-resolution anoscopy-assisted biopsy in MSM [11]. Thus, we have observed that the predictive value of high-grade squamous intraepithelial lesions on anal cytology for high-grade anal intraepithelial neoplasia is limited but consistent, regardless of whether the HPVrelated disease is subclinical or severe enough to merit surgical attention.

Several factors limit our study. Because our cohort consisted of referrals for severe condylomatous disease, the pathological associations may not reflect what exists in MSM with smaller burdens of anogenital condylomata. In addition, only one-third of the subjects had high-resolution anoscopy as part of their evaluation. If routine use of high-resolution anoscopy was more likely to result in surgical referrals having a higher likelihood of high-grade anal intraepithelial neoplasia, the prevalence of high-grade anal intraepithelial neoplasia in this population would be underestimated. However, it should be emphasized that operative excision and pathological analysis without routine preoperative high-resolution anoscopy in the majority of the cohort still demonstrated a high prevalence of high-grade anal intraepithelial neoplasia. Associations taking into account racial demographics, smoking, or type of highly active antiretroviral therapy were not captured by our study. Although the age difference between the HIV-seropositive and HIV-seronegative groups may have favored a higher proportion of high-grade anal intraepithelial neoplasia in the HIV-seropositive group, the EXPLORE study did not show age to be a significant factor for the carriage of high-risk HPV types in HIV-seronegative MSM [12]. Finally, if a fundamental difference exists in the development of high-grade anal intraepithelial neoplasia depending on the location of the condylomatous lesion, then detection bias could have influenced our results, because the exact origin of the histopathological specimen (ie, internal anal vs perianal) was not available.

The present study demonstrates that, in a large urban population of MSM, condylomata requiring surgical excision frequently harbored occult high-grade anal intraepithelial neoplasia or anal squamous cell cancer. These data emphasize the importance of obtaining tissue for histopathological examination in MSM presenting for treatment of anogenital condylomata. Prevention of recurrences and careful clinical follow-up of anal condylomata harboring high-grade anal intraepithelial 
neoplasia may be a method of anal cancer prevention in MSM, particularly in those with HIV infection.

\section{Acknowledgments}

Financial support. H.P.S. was supported by a Ruth L. Kirschstein National Research Service Award (Institutional Research Training Grant T32) and a Bristol-Myers Squibb Virology Fellows Research Training Program Fellowship. L.A.P. was funded in part by a Center for AIDS Clinical Research Pilot Project.

Potential conflicts of interest. L.A.P. reports that she received honoraria from Merck as a member of the speakers' bureau. All other authors: no conflicts.

\section{References}

1. Chin-Hong PV, Palefsky JM. Natural history and clinical management of anal human papillomavirus disease in men and women infected with human immunodeficiency virus. Clin Infect Dis 2002;35:1127-1134.

2. Brown DR, Schroeder JM, Bryan JT, Stoler MH, Fife KH. Detection of multiple human papillomavirus types in condylomata acuminata lesions from otherwise healthy and immunosuppressed patients. J Clin Microbiol 1999; 37:3316-3322.

3. Nyitray A, Nielson CM, Harris RB, Flores R, et al. Prevalence of and risk factors for anal human papillomavirus infection in heterosexual men. J Infect Dis 2008; 197:1676-1684.

4. Bower M, Powles T, Newsom-Davis T, et al. HIV-associated anal cancer: has highly active antiretroviral therapy reduced the incidence or improved the outcome? JAIDS 2004; 37:1563-1565.

5. McCloskey JC, Metcalf C, French MA, Flexman JP, Burke V, Beilin LJ. The frequency of high-grade intraepithelial neoplasia in anal/perianal warts is higher than previously recognized. Int J STD AIDS 2007; 18 : 538-542.

6. Solomon D, Davey D, Kurman R, et al; Forum Group Members; Bethesda 2001 Workshop. The 2001 Bethesda System: terminology for reporting results of cervical cytology. JAMA 2002; 287:2114-2119.

7. Gal AA, Meyer PR, Taylor CR. Papillomavirus antigens in anorectal condyloma and carcinoma in homosexual men. JAMA 1987; 257:337340.

8. Goldstone SE, Winkler B, Ufford LJ, Alt E, Palefsky JM. High prevalence of anal squamous intraepithelial lesions and squamous-cell carcinoma in men who have sex with men as seen in a surgical practice. Dis Colon Rectum 2001;44:690-698.

9. Metcalf AM, Dean T. Risk of dysplasia in anal condyloma. Surgery 1995; 118:724-726.

10. Anderson CA, Boller AM, Richardson CJ, et al. Anal condyloma: A comparison between HIV positive and negative patients. Am Surg 2004; 70 : 1014-1018.

11. Panther LA, Wagner K, Proper J, et al. High resolution anoscopy findings for men who have sex with men: inaccuracy of anal cytology as a predictor of histologic high-grade anal intraepithelial neoplasia and the impact of HIV serostatus. Clin Infect Dis 2004; 38:1490-1492.

12. Chin-Hong PV, Vittinghoff E, Cranston RD, et al. Age-specific prevalence of anal human papillomavirus infection in HIV-negative sexually active men who have sex with men: the EXPLORE study. J Infect Dis 2004; 190:2070-2076. 